\title{
Physical restraint use rate and total fall and injurious fall rates: An exploratory study in two US acute care hospitals
}

\author{
Huey-Ming Tzeng ${ }^{1}$, Chang-Yi Yin ${ }^{2}$ \\ ${ }^{1}$ College of Nursing, Washington State University, Spokane, Washington, USA \\ ${ }^{2}$ Department of History, Chinese Culture University, Taipei, Taiwan, China \\ Email: tzenghm@gmail.com
}

Received 7 June 2012; revised 6 July 2012; accepted 17 July 2012

\begin{abstract}
This exploratory study used archived hospital data to investigate the relationships between the percentage of patients with physical restraints and the total fall rate as well as the injurious fall rate per 1000 patientdays. The two tested research questions were 1) What is the relationship between the restraint use rate and the total fall rate? 2) What is the relationship between the restraint use rate and the injurious fall rate? The results showed that a higher restraint use rate was associated with a higher total fall rate, yet a lower injurious fall rate in adult inpatient acute care settings. In efforts for fall and injurious fall prevention, front-line managers need to balance the frequency and appropriateness of physical restraint use with optimizing patients' physical activity. Future research should explore the cause-effect relationship between physical restraint use and consequent injurious fall incidents.
\end{abstract}

Keywords: Accidental Falls; Hospital-Acquired Injury; Hospital; Nurse; Nursing; Patient Safety; Physical Restraint

\section{INTRODUCTION}

Previous literature and current US regulations stipulate that hospitals should restrict the use of physical restraints [1]. Centers for Medicare \& Medicaid Services [1] have also set a standard related to physical restraint use, which stresses that "...the patient has the right to be free from restraints of any form that are not medically necessary...” [1, p. 81]. For hospitalized patients, “...the use of restraints should be frequently evaluated and ended at the earliest possible time based on the assessment and reevaluation of the patient's condition..." [1, p. 94]. Additionally, hospitals have been urged to minimize or avoid physical restraints to prevent adult inpatient falls and related injuries [2]. The study conducted by Tiessen and associates [3] showed that the transformation of the patient safety culture in a Canadian community hospital to a restraint-free physical environment resulted in an inpatient injurious fall rate that was lower than the industry average. However, other studies $[4,5]$ continued to show that clinicians seem to be conflicted about the role of physical restraints in fall management in both acute and long-term inpatient care settings. Applying alternatives to physical restraints in inpatient care settings has become commonly accepted as part of standard nursing practice [4].

Many extrinsic and intrinsic patient risk factors may lead to falls and injurious falls during hospital stays [6]. A number of fall prevention interventions have been proposed or are available, but most of the interventions have never been adequately tested [6-8]. For example, Inglesby [9] proposed an A-B-C approach to prevent falls in hospital settings. " $\mathrm{A}$ " stands for the fall prevention interventions related to administrative initiatives and assessment for risk factors; " $\mathrm{B}$ " is for the interventions related to balance, bed, and bathroom; and " $\mathrm{C}$ " stands for color-coding the level of risk that a patient has for falling. Clyburn and Heydemann's [10] analysis and comprehensive review of fall prevention methods used in hospitals concluded that data are insufficient for evidence-based guidelines being effective in fall prevention. They claimed that many of the interventions are based on expert opinion and statistical trends [10]. To the clinicians who deliver the bedside care, there is a desperate need to clarify whether restraint use may lead to falls and injurious falls or whether it is useful in fall and injurious fall prevention.

\section{Purpose of the Study}

This exploratory study used archived hospital data to investigate the relationships between the percentage of patients with physical restraints (limbs and/or vest) and the total fall rate as well as the injurious fall rate per 1000 patient-days. This study was conceptualized based 
on Donabedian's framework of structure, process, and health-care outcomes [11]. Donabedian suggested that structure and process factors are associated with the outcome of health-care organizations. Therefore, the present study proposed the total fall rate and the injurious fall rate as two patient safety outcome indicators and the restraint use rate as a process indicator.

Two tested research questions were 1) What is the relationship between the restraint use rate and the total fall rate? and 2) What is the relationship between the restraint use rate and the injurious fall rate?

\section{RESEARCH METHODS}

\subsection{Design}

This exploratory study was conducted at two hospitals located in Michigan in the United States. Archived hospital data was used for analysis. This study was approved by each hospital's institutional review board (IRB) and the IRB of the correspondent author's employer university. There was no conflict of interest.

\subsection{Sample and Data Sources}

A convenience sample of adult inpatient acute care units from the two hospitals was included. A total of 10 adult medical, surgical, and medical-surgical combined inpatient acute care units provided archived hospital data and reports. One study site was a community hospital with about 300 beds and four units (two medical units, one surgical unit, one medical-surgical unit; February 2007December 2008), and the other one was a teaching medical center with 700 beds and six units (two medical units, two surgical units, and two medical-surgical units; October 2006-December 2008).

Patient care unit-month (abbreviated as unit-month) was the unit of analysis, which was defined as data aggregated by month for each patient care unit. For example, the acute surgical unit (52 beds in operation) of the community hospital had monthly fall rate data from February 2007 to December 2008. This acute surgical unit would have a total of 23 unit-month data points. We were aware that some interdependence for the data points from a single unit and for the data points from other units in the same hospital existed. In this study, each data point for a study unit was assumed to be independent from all others. All 23 unit-month data points from the acute surgical unit were included in the analysis as 23 cases.

In this study, the total sample size in unit-month was calculated as the total months with available data for each patient care unit. In other words, a total of 220 data points (92 from the community hospital and 128 from the teaching medical center) were included in the data analysis.
It is noted that the patients included in the calculation of the aggregated unit-month values (the restraint use rates, the total fall rates, and the injurious fall rates) were not necessarily the ones who had restraints during hospital stays. The operational definitions of study variables follow.

\subsection{Fall and Injurious Fall Rates}

Archived patient fall incidents and patient-day data for the study units during the study periods were retrieved by the hospital administrators and then forwarded to the corresponding author. A fall was defined as an unplanned descent to the floor, with or without injury. The total fall rate was defined as the number of patient falls (including those with or without injury) during hospital stays per 1000 patient-days. The injurious fall rate was defined as the fall rate per 1000 inpatient-days for which physical injury occurred, regardless of the severity $[12,13]$.

As for the operational definitions, the total fall rate was calculated by unit-month: (number of total falls $x$ 1000)/(total patient-days). The injurious fall rate was calculated by unit-month: (number of injury falls $\times 1000$ )/ (total patient-days).

As an example, the total fall rate for March 2007 at the acute surgical unit of the community hospital was calculated based on the total number of patient fall incidents and the total number of patient-days at this acute surgical unit during March 2007. The total patient-days for March 2007 were the sum of the daily count of patient-days. The daily count of patient-days was the count of patients at midnight who were officially admitted to the unit and were not officially discharged or transferred; this information was captured by each hospital's bed management system. For instance, a $100 \%$ bed occupancy rate for each day of March 2007 would result in a total of 1612 patient-days ( 52 beds $\times 31$ days). As an illustration for how the total fall rate was calculated, the acute surgical unit of the community hospital had a $100 \%$ bed occupancy rate for each day in March 2007 (for a total of 1612 patient-days) and two patient falls. The fall rate for March 2007 would be 1.24 per 1000 patient-days: (2 falls $\times 1000) /(1612$ patient-days $)$.

\subsection{Restraint Use Rate}

Archived restraint prevalence data for the study units during the study periods was retrieved by the hospital administrators and then forwarded to the corresponding author. The restraint prevalence data were previously collected and prepared for the National Database of Nursing Quality Indicators [13]. The restraint use rate was a restraint prevalence of all inpatients with a limb and/or vest restraint in use on the day of prevalence study at each study unit. The prevalence study was con- 
ducted by designated hospital administrators/staff on one specified day every 3 months (e.g., the second Wednesday of each quarter) [13].

The definition of the restraint use rate adopted in this study was the same as in The National Database of Nursing Quality Indicators: Description \& Glossary [13], which was used by the study hospitals.

For the operational definition, the restraint use rate on the day the prevalence study was conducted (every 3 months) was calculated by unit: (number of patients with a limb and/or vest restraint) $\times 100 /$ (total number of surveyed patients). The restraint use rate was quarterly.

\subsection{Data Analyses}

The SPSS 19.0 Windows version (SPSS Inc., Chicago, IL, USA) was used. Prior to conducting data analyses, the restraint use rate for each study unit and quarter was matched with the fall and injurious fall rates for the corresponding unit and corresponding months. Pearson product-moment correlation analyses were used to answer two research questions. Alpha was set at 0.05 .

\section{RESULTS}

\subsection{Descriptive Analyses}

The average total fall rate was $3.61(\mathrm{SD}=2.59$; range: 0 - 18.21) and the average injurious fall rate was 0.72 (SD $=1.05$; range: $0-5.44)$. The average restraint use rate was $2.61 \%(\mathrm{SD}=4.39$; range: $0 \%-16.28 \%)$.

For exploratory purposes, independent t-tests were conducted to explore the impact of the hospital (the community hospital vs. the teaching medical center) on the study variables (Table 1). The independent $t$-tests showed that there was no significant difference in the total fall rates between the two hospitals. The community hospital had a significantly lower injurious fall rate and a higher physical restraint use rate than the teaching medical center. Alpha was set at 0.05 . One-way analyses of variance (ANOVA) were also conducted between groups to explore the impact of the three unit types (the medical unit, surgical unit, medical-surgical unit) on the study variables (Table 2). The one-way ANOVA tests showed that there was no significant difference in the total fall rates and injurious fall rates across the three unit types. However, there was a statistically significant difference in the restraint use rate for the three unit types. Post hoc comparisons using the Tukey HSD test indicated that the mean restraint use rate for the surgical units was signifycantly lower than those for the medical units and the medical-surgical units. The mean restraint use rate for the medical units did not differ significantly from that of the medical-surgical units. Alpha was set at 0.05 .

To better understand the characteristics of the study hospitals and units, for exploratory purposes, the means of the length of stay (the total patient-days for the month divided by the number of patients discharged from the unit for the month) were also investigated. Including all 10 study units, the average length of stay was 4.71 days $(\mathrm{SD}=1.01$; range: $3.10-8.56)$. For the four study units of the community hospital, the average length of stay was 4.47 days ( $\mathrm{SD}=0.75$; range: $3.10-5.85$ ). For the six study units of the teaching medical center, the average length of stay was 4.89 days ( $\mathrm{SD}=1.14$; range: 3.22 8.56). An independent t-test showed that the community hospital had a significantly lower average length of stay than the teaching medical center (Table 1). Among the four medical units, the average length of stay was 4.97 days ( $\mathrm{SD}=1.35$; range: $3.10-8.56)$. Among the three surgical units, the average length of stay was 4.56 days $(\mathrm{SD}=0.70$; range: $3.57-6.51)$. Among the three medical-surgical units, the average length of stay was 4.52 days $(\mathrm{SD}=0.71$; range: $3.22-5.85)$. The one-way ANOVA test showed that there was a statistically significant difference in the average length of stay in days for the three unit types. Post hoc comparisons using the Tukey HSD test indicated that the mean length of stay

Table 1. The summary of the results of independent t-tests: the impact of hospitals.

\begin{tabular}{|c|c|c|c|c|c|c|c|}
\hline & Hospital Type & $\mathrm{N}$ & Mean & SD & $\mathrm{t}$ & $\mathrm{df}$ & Sig. (2-tailed) \\
\hline \multirow[b]{2}{*}{ Total fall rate per 1000 patient-days } & Community hospital & 92 & 3.87 & 2.13 & \multirow[b]{2}{*}{1.27} & \multirow[b]{2}{*}{218} & \multirow[b]{2}{*}{0.21} \\
\hline & Teaching medical center & 128 & 3.42 & 2.87 & & & \\
\hline \multirow{2}{*}{$\begin{array}{l}\text { Injurious fall rate per } 1000 \\
\text { patient-days }\end{array}$} & Community hospital & 92 & 0.36 & 0.68 & \multirow{2}{*}{-4.94} & \multirow{2}{*}{209.35} & \multirow{2}{*}{$\mathrm{P}<0.01$} \\
\hline & Teaching medical center & 128 & 0.98 & 1.18 & & & \\
\hline \multirow{2}{*}{$\begin{array}{c}\% \text { of patients with physical } \\
\text { restraints }\end{array}$} & Community hospital & 92 & 5.98 & 5.05 & \multirow{2}{*}{10.88} & \multirow{2}{*}{95.55} & \multirow{2}{*}{$\mathrm{P}<0.01$} \\
\hline & Teaching medical center & 128 & 0.19 & 0.94 & & & \\
\hline \multirow{2}{*}{ Average length of stay in days } & Community hospital & 92 & 4.47 & 0.75 & \multirow{2}{*}{-3.27} & \multirow{2}{*}{212.62} & \multirow{2}{*}{$\mathrm{P}<0.01$} \\
\hline & Teaching medical center & 125 & 4.89 & 1.14 & & & \\
\hline
\end{tabular}


Table 2. The summary of the results of one-way ANOVA tests: the impact of unit types.

\begin{tabular}{|c|c|c|c|c|c|c|c|}
\hline & Unit Type & $\mathrm{N}$ & \multicolumn{2}{|c|}{ Mean } & \multicolumn{3}{|c|}{ SD } \\
\hline \multirow{4}{*}{ Total fall rate per 1000 patient-days } & Medical unit & 82 & \multicolumn{2}{|c|}{3.50} & \multicolumn{3}{|c|}{2.26} \\
\hline & Surgical unit & 88 & \multicolumn{2}{|c|}{3.56} & \multicolumn{3}{|c|}{3.05} \\
\hline & Medical-surgical & 50 & \multicolumn{2}{|c|}{3.87} & \multicolumn{3}{|c|}{2.21} \\
\hline & Total & 220 & \multicolumn{2}{|c|}{3.61} & \multicolumn{3}{|c|}{2.59} \\
\hline \multirow{4}{*}{ Injurious fall rate per 1000 patient-days } & Medical unit & 82 & \multicolumn{2}{|c|}{0.65} & \multicolumn{3}{|c|}{0.84} \\
\hline & Surgical unit & 88 & \multicolumn{2}{|c|}{0.77} & \multicolumn{3}{|c|}{1.17} \\
\hline & Medical-surgical & 50 & \multicolumn{2}{|c|}{0.74} & \multicolumn{3}{|c|}{1.12} \\
\hline & Total & 220 & \multicolumn{2}{|c|}{0.72} & \multicolumn{3}{|c|}{1.05} \\
\hline \multirow{4}{*}{$\%$ of patients with physical restraints } & Medical unit & 82 & \multicolumn{2}{|c|}{3.33} & \multicolumn{3}{|c|}{4.87} \\
\hline & Surgical unit & 88 & \multicolumn{2}{|c|}{1.28} & \multicolumn{3}{|c|}{2.98} \\
\hline & Medical-surgical & 50 & \multicolumn{2}{|c|}{3.79} & \multicolumn{3}{|c|}{5.08} \\
\hline & Total & 220 & \multicolumn{2}{|c|}{2.61} & \multicolumn{3}{|c|}{4.39} \\
\hline \multirow{5}{*}{ Average length of stay in days } & Medical unit & 82 & & & & 35 & \\
\hline & Surgical unit & 85 & & & & & \\
\hline & Medical-surgical & 50 & & & & 71 & \\
\hline & Total & 217 & & & & 01 & \\
\hline & ANOVA & Sum of Squares & $\mathrm{df}$ & Mean Square & $\mathrm{F}$ & & Sig. \\
\hline & Between groups & 4.59 & 2 & 2.30 & 0.34 & & 0.71 \\
\hline Total fall rate per 1000 patient-days & Within groups & 1463.38 & 217 & 6.74 & & & \\
\hline & Total & 1467.97 & 219 & & & & \\
\hline & Between groups & 0.70 & 2 & 0.35 & 0.32 & & 0.73 \\
\hline Injurious fall rate per 1000 patient-days & Within groups & 238.58 & 217 & 1.10 & & & \\
\hline & Total & 239.28 & 219 & & & & \\
\hline & Between groups & 267.48 & 2 & 133.74 & 7.33 & & $\mathrm{P}<0.01$ \\
\hline$\%$ of patients with physical restraints & Within groups & 3961.18 & 217 & 18.25 & & & \\
\hline & Total & 4228.66 & 219 & & & & \\
\hline & Between groups & 9.39 & 2 & 4.69 & 4.74 & & 0.01 \\
\hline Average length of stay in days & Within groups & 211.81 & 214 & 0.99 & & & \\
\hline & Total & 221.20 & 216 & & & & \\
\hline
\end{tabular}

for the medical units was significantly longer than the ones for the surgical units and the medical-surgical units. The mean length of stay for the surgical units did not differ significantly from the one for the medical-surgical units (Table 2). Alpha was set at 0.05 .

\subsection{Correlation Analyses}

Pearson correlation analyses showed that high restraint use rates were associated with high total fall rates $(r=$
$0.15, \mathrm{n}=220, \mathrm{P}=0.02)$. In other words, if the physical restraints (a lower restraint use rate) were not used, the total fall rates would be lower. In contrast, high restraint use rates were associated with low injurious fall rates ( $\mathrm{r}$ $=-0.20, \mathrm{n}=220, \mathrm{P}=0.003$ ).

\subsection{Additional Analyses}

For exploratory purposes only, we conducted Pearson correlation analyses on the relationship of the average 
length of stay with the total fall rate, injurious fall rate, and restraint use rate. The correlation analyses showed that the average length of stay was statistically signifycantly associated with the injurious fall rate $(\mathrm{r}=0.15, \mathrm{n}=$ $217, P=0.03$ ). The average length of stay was not found to be associated with the total fall rate or restraint use rate.

Two multiple regression analyses were also conducted with the total fall rate and injurious fall rate as the dependent variable for each model. The restraint use rate as the predictor after controlling for the hospital (one dummy variable to differentiate the community hospital from the teaching medical center), unit types (two dummy variables to capture three unit types), and average length of stay in days (a continuous variable). For the multiple regression model of the total fall rate as the dependent variable, none of the predictor or control variables was statistically significant. The multiple regression model of the injurious fall rate showed that only the hospital variable was statistically significant; the community hospital had a significantly lower injurious fall rate than the teaching medical center, and this conclusion is consistent with the finding of the independent t-test. The detailed results of the multiple regression analyses can be obtained from the correspondent author.

\section{DISCUSSION}

Both research questions were answered using Pearson correlation analyses. For the first research question (What is the relationship between the restraint use rate and the total fall rate?), we found that a higher restraint use rate was associated with a higher total fall rate. This finding is consistent with the recommendation of Hartford Institute for Geriatric Nursing [2]. In addition, it is possible that a higher restraint use rate would lead to increased frequency of nursing documentations and surveillance at bedside in responding to the orders of physical restraint use. Due to increased surveillance at bedside, falls without injuries by patients in physical restraints and by their roommates may be more easily observed by nursing staff. Consequently, more falls may be documented when the unit has a higher restraint use rate. Additional research is warranted to validate this inference.

For the second research question (What is the relationship between the restraint use rate and the injurious fall rate?), the findings suggested that greater restraint use was associated with fewer fall-related injuries. This conclusion challenges the current restraint use practice recommendation as entailed in a previous study [3] and stipulated by US regulations [1] that in efforts for injurious fall prevention, hospitals should restrict the use of physical restraints. More extensive research is needed to validate whether restraints could lead to decreased sever- ity of fall-related injuries. Future research should investtigate whether a patient fell and then had a restraint applied or the patient had a restraint and then fell.

The additional analyses also provided some insights. The average length of stay was found to be positively associated with the injurious fall rate $(\mathrm{r}=0.15, \mathrm{P}=0.03)$. However, it is arguable whether a longer average length of stay would lead to a higher injurious fall rate in adult acute medical, surgical, and medical-surgical units. More research is warranted to validate whether having an injurious fall during hospitalization could lead to an increased length of stay or, vice versa, whether having a longer length of hospital stay could lead to an increased chance of having an injurious fall.

Our study may be limited by some interdependence existing for the data from the same unit and from the same hospital. This study cannot be used to conclude that a high restraint rate would lead to a high fall rate and a low injurious fall rate because the cause-effect relationship was not investigated. The reason is that the prevention of falls could be one explanation for the use of restraints. Also, while the correlation coefficients between restraint use rates and total fall rates $(r=0.15)$ and between restraint use rates and injurious fall rates $(r=$ -0.20 ) are low, this study did not include information regarding correlations with possible confounding variables such as mobility and cognitive status.

\section{CONCLUSION AND PRACTICAL IMPLICATIONS}

We concluded that the restraint use rate was linked to the fall and injurious fall rates in adult inpatient acute care settings; a higher restraint use rate was found to be associated with a higher total fall rate and with a lower injurious fall rate. The restraint use rate was conceptualized as a process indicator, which may be a point of intervention to improve patient outcomes (the total fall rate and the injurious fall rate). Therefore, in efforts for fall and injurious fall prevention, front-line managers need to balance the frequency and appropriateness of physical restraint use with optimizing patients' physical activity.

For nursing administrators, it is essential to address fall prevention policies after having a thorough understanding of the practice culture (e.g., restraint use practice by type of specialty, clinicians' knowledge, attitudes and behaviors related to restrain use) and taking into account the differences across unit types and characteristics (e.g., average length of stay). As found in this study, the average length of stay was found to be positively associated with the injurious fall rate. In addition, the mean length of stay for the medical units was significantly longer than the ones for the surgical units and the medical-surgical units. Also, the mean restraint use rate for 
the surgical units was significantly lower than those for the medical units and the medical-surgical units. Similar to the A-B-C approach to prevent falls described by Inglesby [9], development or revision of administration policies in relation to either or both fall prevention and restraint use should start by 1) identifying problems; 2) communicating the problems to the stakeholders (e.g., clinicians, patients and their family members); 3) taking actions and committing to adopt change; and 4) sustaining an improvement process.

Clinical researchers may use prospective research designs to explore the cause-effect relationship between patients' physical restraint use and consequent fall and injurious fall incidents. It is essential to use both individual patient-level and patient care unit-level data. Unitlevel data may reflect factors such as the culture of patient safety related to restraint use and fall management. Including possible confounding variables (e.g., patient mobility, cognitive status, and hearing problem) in the analysis is warranted for developing patient-centered injurious fall management strategies. It is also important to take into account the differences in the cultures of fall prevention practice and restraint use. As found in this study, the community hospital had a significantly lower injurious fall rate and a higher physical restraint use rate than the teaching medical center. Multi-hospital studies are warranted.

\section{ACKNOWLEDGEMENTS}

This project was supported by grant number R03HS018258 from the Agency for Healthcare Research and Quality. The content is solely the responsibility of the authors and does not necessarily represent the official views of the Agency for Healthcare Research and Quality.

\section{REFERENCES}

[1] Centers for Medicare \& Medicaid Services (2012) State operations manual: Appendix A-Survey protocol, regulations and interpretive guidelines for hospitals. www.gnyha.org/2802/File.aspx

[2] Hartford Institute for Geriatric Nursing (2008) Preventing falls in acute care. In: Capezuti, E., Zwicker, D. Mezey, M. and Fulmer, T., Eds., Evidence-Based Geriatric Nursing Protocols for Best Practice, 3rd Edition. Springer Publishing Company, New York, 161-198.
[3] Tiessen, B., Deter, C., Snowdon, A. and Kolga, C. (2010) Continuing the journey to a culture of patient safety: From falls prevention to falls management. Healthcare Quarterly, 13, 79-83.

[4] McCabe, D.E., Alvarez, C.D., McNulty, S.R. and Fitzpatrick, J.J. (2011) Perceptions of physical restraints use in the elderly among registered nurses and nurse assistants in a single acute care hospital. Geriatric Nursing, 32, 39-45. doi:10.1016/i.gerinurse.2010.10.010

[5] Williams, J., Kaasalainen, S., Hadjistavropoulos, T., Scudds, R., Thorpe, L., Neville, S., Tremeer, J. and Andersen, D. (2011) A qualitative investigation of injurious falls in long-term care: Perspectives of staff members. Disability and Rehabilitation, 33, 423-432. doi: $10.3109 / 09638288.2010 .498555$

[6] Currie, L. (2008) Fall and injury prevention. In: R. G. Hughes, Ed., Patient Safety and Quality: An EvidenceBased Handbook for Nurses, Agency for Healthcare Research and Quality, US Department of Health and Human Services, Rockville, 195-250.

[7] Gray-Micelli, D. (2008) Preventing falls in acute care. In: Capezuti, E., Zwicker, D., Mezey, M. and Fulmer, T. Eds., Evidence-Based Geriatric Nursing Protocols for Best Practice, 3rd Edition, Springer Publishing, New York, 161-198.

[8] Joint Commission (2005) Strategies for addressing the root causes of falls. In: Smith, I.J., Ed., Reducing the Risk of Falls in Your Health Care Organization, Joint Commission, Oakbrook Terrace, 29-50.

[9] Inglesby, T. (2012) Preventing falls: The A-B-C approach. PSQH: Patient Safety \& Quality Healthcare, 9, 46-51.

[10] Clyburn, T.A. and Heydemann, J.A. (2011) Fall prevention in the elderly: Analysis and comprehensive review of methods used in the hospital and in the home. Journal of the American Academy of Orthopaedic Surgeons, 19, 402-409.

[11] Donabedian, A. (1986) Criteria and standards for quality assessment and monitoring. Quality Research Bulletin, 12, 99-108.

[12] National Quality Forum (2009) Safety practice 33: Falls prevention. In: Safe Practices for Better Healthcare2009 Update: A Consensus Report, National Quality Forum, Washington DC, 319-324.

[13] The American Nurse Association, Inc. (ANA) (2009) The national database of nursing quality indicators: Description \& glossary. American Nurses Association, Inc., Silver Spring. 